\title{
Pobreza multidimensional en Chiapas: generalizadA PERO HeterogÉNEA
}

\author{
Multidimensional Poverty in Chiapas: Widespread but Heterogeneous
}

\author{
Alma Esther Aguilar-Estrada \\ Ignacio Caamal-Cahuich \\ Miguel Ángel Ortiz-Rosales
}

Resumen: Aunque Chiapas ha sido históricamente un estado prioritario en combate a la pobreza, el 76\% de la población persiste en esta situación. El objetivo de este trabajo es distinguir perfiles de pobreza a nivel municipal para la entidad y proponer políticas públicas diferenciadas. La metodología combina análisis multivariado y sistemas de información geográfica. Se obtuvieron cuatro conglomerados o perfiles de pobreza que se presentan con mapas y propuestas de política pública diferenciadas. Se concluye que la pobreza continúa siendo un tema de interés prioritario en Chiapas, y que para lograr resultados a largo plazo es necesario considerar las particularidades de cada perfil y priorizar acciones particulares.

Palabras clave: bienestar, derechos sociales, pobreza, políticas públicas.

Abstract: The state of Chiapas has historically been a priority target in the fight against poverty. Currently, however, $76 \%$ of the population continues to be impoverished. The objective of this paper is to distinguish poverty profiles at the municipal level and propose differentiated publicpolicies. The methodology combines multivariate analysis and geographic information systems. We obtained four composites or poverty profiles, which are presented with maps and differentiated public policy proposals. We conclude that given the intensity of poverty in Chiapas, it should continue to be a priority for poverty-reduction efforts. Yet to achieve results in the long term, the particularities of each profile should be considered, since in each case different measures may have greater priority.

Keywords: welfare, social rights, poverty, public policies.

\footnotetext{
Alma Esther Aguilar Estrada. Doctora en Economía por el Colegio de Postgraduados, México. Posdoctorante en la División de Ciencias Económico-Administrativas de la Universidad Autónoma Chapingo. México. Temas de especialización: combate a la pobreza y estudios de política pública y de prospectiva. Correo electrónico: libelunazul@gmail.com.

Ignacio Caamal Cahuich. Doctor en Economía por la Universidad Autónoma Chapingo, México. Profesor-investigador en la División de Ciencias Económico-Administrativas de la Universidad Autónoma Chapingo, México. Temas de especialización: economía y administración de empresas agropecuarias, política agrícola y desarrollo rural. Correo electrónico: icaamal82@yahoo.com.mx.
}

Miguel Ángel Ortiz Rosales. Doctor en Economía por el Colegio de Postgraduados, México. Consultor en el Colegio de Postgraduados, México. Temas de especialización: economía del conocimiento y estudios de política pública y de prospectiva. Correo electrónico: maor_91@yahoo.com.mx.

Enviado a dictamen: 28 de noviembre de 2017 Aprobación: 12 de marzo de 2018 Revisiones: 1 


\section{Introducción}

$\mathrm{D}$ urante años, los organismos internacionales han adoptado el método del ingreso mínimo o línea de bienestar para medir la pobreza, principalmente porque permite hacer comparativos entre países sólo con contrastar los umbrales económicos con el nivel de ingreso de las familias (Townsend, 1993; Olinto et al., 2013).

En el debate económico actual para definir la pobreza se ha incorporado la dimensión de los derechos sociales y se resalta que no es suficiente contar con un nivel determinado de ingresos, sino que deben cubrirse necesidades de manera permanente para que cada persona tenga la posibilidad de desarrollar capacidades. De esta manera, en la actualidad la pobreza se reconoce como un problema multidimensional (Sen, 1991 y 2003; Alkire y Foster, 201l; Alkire, Conconi y Seth, 2014).

México fue el primer país del mundo en contar con un sistema de medición oficial multidimensional de la pobreza (CONEVAL, 2010). Hoy en día, para medir la pobreza en este país se consideran tanto el ámbito del bienestar, a partir del nivel de ingresos, como el ámbito de los derechos sociales, a partir de la medición de seis variables: rezago educativo, acceso a la alimentación, acceso a la salud, acceso a la seguridad social, calidad y espacios de la vivienda y servicios básicos de la vivienda. Esta metodología ha brindado la posibilidad de desagregar la información para contrastar entidades, municipios y carencias principales, lo que permite elaborar propuestas de política pública con un mejor nivel de precisión al momento de focalizar programas.

A partir de esta metodología, las estadísticas más recientes reflejan que en México el 46.2\% de la población es pobre multidimensional y el $9.5 \%$ se encuentra en pobreza multidimensional extrema. La situación es mucho más grave en Chiapas que en cualquier otra entidad porque en este estado el $77 \%$ de la población se encuentra en pobreza multidimensional y el $28 \%$ en pobreza multidimensional extrema; además de ello, la tendencia es creciente en los últimos años (CONEVAL, 2017). La incidencia muestra que la pobreza extrema en Chiapas triplica la media nacional.
Sin embargo, la situación de pobreza en el estado no es un problema reciente, porque durante las últimas décadas se ha observado que su incidencia e intensidad son bastante elevadas en comparación con las del resto del país, que ambas dimensiones se encuentran fuertemente relacionadas y que se acentúan al existir un nivel de desigualdad muy por encima del promedio nacional (Cortés et al., 2007; Aguilar, 2016).

Ante esta tendencia, y principalmente después del levantamiento del Ejército Zapatista de Liberación Nacional en enero de 1994, Chiapas ha sido un estado prioritario para la intervención de programas sociales. No obstante, éstos han sido ampliamente criticados, pues además de utilizarse para "superar" sólo en apariencia los indicadores de pobreza, se han convertido en mecanismos de manipulación política de los más pobres, y en medios para mantener clientelas políticas y ejercer prácticas de cacicazgo y corrupción por parte de los encargados de su implementación (Lerner, 1996; Dresser, 1997; Gordon, 2004; Rodríguez, 2007).

Por otro lado, cuando la pobreza se convierte en un problema generalizado, la acción de la política pública debe centrarse en generar condiciones que permitan una igualdad de capacidades básicas (Sen, 2003), lo que hace necesarios los programas sociales. Ahora bien, diversos autores han mostrado que la política social produce mejores resultados cuando la pobreza se analiza desde un enfoque multidimensional y las propuestas se llevan a cabo a partir de una adecuada complementariedad entre focalización y universalización (Brodersohn, 1999; Alemayehu et al., 2005; Backiny-Yetna y Wodon, 2009).

En el presente trabajo se pretende caracterizar la pobreza multidimensional en el estado de Chiapas, distinguir los tipos e intensidades de pobreza a nivel municipal y proponer una agenda pública para el estado acorde a las necesidades particulares de la población y a las prioridades en cada uno de sus municipios.

\section{Metodología}

Se conformó una base de datos con los resultados de pobreza multidimensional a nivel municipal para los 118 municipios del estado de Chiapas a partir de las 
estimaciones del Consejo Nacional de Evaluación de la Política de Desarrollo Social (CONEVAL), con base en el Módulo de Condiciones Socioeconómicas de la Encuesta Nacional de Ingresos y Gastos de los Hogares (MCS-ENIGH 2010) y la muestra del Censo de Población y Vivienda elaborados por el Instituto Nacional de Geografía y Estadística (INEGI). Se incluyó cada una de las variables de bienestar y de derechos sociales de acuerdo con el método de medición establecido en los "Lineamientos y criterios generales para la definición, identificación y medición de la pobreza" (CONEVAL, 2009).

El bienestar se mide a partir de la variable "ingreso", representada con el monto promedio de ingresos por hogar en cada municipio. Los derechos sociales se miden con seis variables: servicios básicos, calidad y espacios de la vivienda, alimentación, educación, salud y seguridad social, indicándose el porcentaje de hogares sin acceso a cada una de estas variables. La base de datos se representó de forma matricial en el programa Excel, con 118 municipios como filas y siete variables como columnas. La expresión matemática de la matriz de pobreza (P) es la siguiente:

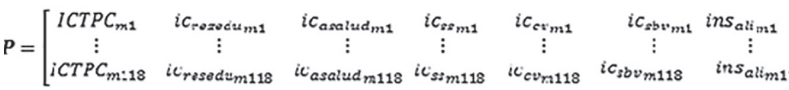

En la cual:

$P=$ Matriz de indicadores municipales de pobreza multidimensional en Chiapas. $I C T P C_{m}=$ Ingreso corriente total per cápita promedio municipal .

ic_reced $u_{m}=$ Porcentaje de personas con carencia porrezago educativo en el municipio.

ic_asalud $_{m}=$ Porcentaje de personas con carencia por acceso a servicios de salud en el municipio.

$i c_{-} c v_{m}=$ Porcentaje de personas con carencia de calidad y espacios de la vivienda en el municipio.

ic_sbvm= Porcentaje de personas con carencia por acceso a servicios básicos de la vivienda en el municipio.

ins_alim= Porcentaje de personas con carencia por acceso a la alimentación en el municipio.

Esta matriz se exportó al programa Infostat para proceder con el análisis cluster. Se eligió este tipo de análisis como método estadístico multivariado partiendo de las siguientes premisas: que cada municipio perteneciera a un solo grupo, que todo municipio quedara clasificado y que cada grupo fuera internamente homogéneo, en otras palabras, que se lograra maximizar la varianza inter-grupos y minimizar la varianza dentro de los grupos (Peña, 2002; Montanero, 2008). Se eligió el principio de aglomeración no jerárquico K-means y distancia euclidea al cuadrado, y se dio la instrucción de estandarizar los datos debido a que la variable "ingreso" se encontraba en números absolutos y el resto en relativos. Considerando la importancia de la validación de los resultados como etapa final en la elaboración de conglomerados (Aldenderfer y Blashfield, 1984), los resultados del agrupamiento se validaron por medio del "análisis discriminante" y se renombraron para formar la tipología como municipios de perfiles I, II, III y IV.

La tipología permitió describir las características particulares de cada conglomerado en función de la intensidad de las siete variables analizadas. Sin embargo, dado que el resultado de estas variables de pobreza multidimensional dependía de otras variables para la construcción de cada indicador, se decidió llevar a cabo un segundo nivel de análisis. Para ello, se recurrió a los microdatos de la MCS ENIGH 2010 a fin de analizar la incidencia de las variables que se usan para la construcción de los indicadores de pobreza multidimensional en cada uno de los perfiles obtenidos. Por mencionar un ejemplo, el indicador de "calidad y espacios de la vivienda" para cada perfil permitió obtener un primer nivel de análisis, y el segundo nivel de análisis se logró cruzando los microdatos de la ENIGH con los perfiles obtenidos con las variables de "material de pisos", "material de muros", "material de techos" y "hacinamiento", los cuales se consideran en la construcción del indicador.

Lo anterior permitió contar con un panorama más detallado en la composición de cada indicador de pobreza multidimensional, identificar zonas geográficas prioritarias con base en cada perfil y plantear propuestas de política social con enfoques de atención gubernamental focalizada o universalizada. 


\section{Resultados}

El nivel de todos los indicadores de pobreza multidimensional en Chiapas se encuentra muy por encima del promedio nacional. Los que más contribuyen a la intensidad de la pobreza son: el acceso a servicios básicos, determinado principalmente por la prevalencia de viviendas sin chimenea para cocinar; el rezago educativo, en el que la entidad ocupa el primer lugar nacional; y la carencia por acceso a seguridad social, que se relaciona directamente con el origen del ingreso de los hogares y el nivel de informalidad (ver Figura 1).

Ahora bien, a pesar del nivel generalizado de pobreza en la entidad, Chiapas puede dividirse en cuatro conglomerados que se consideran adecuados para distinguir intensidades de pobreza y prioridades de política pública a nivel municipal (ver Tabla 3 y Mapa 1). La clasificación obtenida con el análisis cluster se sometió a otra técnica multivariada, el análisis discriminante, con la finalidad de minimizar la probabilidad de equivocarse en la asignación de municipios para cada perfil. El análisis de varianza univariado y la prueba de homogeneidad de matrices de covarianzas valida la tipología definitiva, lo que permite la máxima heterogeneidad entre grupos y homogeneidad al interior (ver Tablas 1 y 2).

En un comparativo inicial entre perfiles, la dimensión de bienestar muestra que el perfil más bajo presenta un nivel de ingresos que ni siquiera es suficiente para adquirir una canasta alimentaria rural, considerando el precio de la misma al momento del levantamiento de información del MCS-ENIGH 2010. A medida que aumenta el perfil disminuyen las condiciones de pobreza, pero también cambian las características de la misma tanto por las variables que la determinan, como por el contexto geográfico, económico y cultural de los municipios (ver Tabla 3). A continuación se presentan las particularidades, prioridades y propuestas diferenciadas para cada uno de los conglomerados.

\section{Perfil I. Indígenas, los pobres más extremos}

El primer conglomerado está formado por veintitrés municipios que concentran el 15\% de la población estatal cuya pobreza es tan intensa que los coloca en un primer nivel de prioridad. Se trata de municipios indígenas en su totalidad, ubicados en las regiones de Los Altos y Selva, donde residen personas de las etnias tseltal, tsotsil y chol (ver Mapa 1 y Tabla 6).

La pobreza en estos municipios es tan elevada que el ingreso promedio ni siquiera alcanza la línea de bienestar mínimo rural, es decir, el costo de la canasta básica alimentaria (ver Tabla 3). La situación en la dimensión de derechos sociales es también muy grave; cerca de la mitad de la población carece de acceso al derecho a la alimentación debido a una dieta muy poco variada e insuficiente, limitada a los alimentos que ellos mismos producen o que reciben como apoyos gubernamentales en especie.

La situación de pobreza extrema se refleja en las viviendas, pues más del 90\% carece de servicios básicos (ver Tabla 4) debido a que en la gran mayoría se cocina con leña o carbón sin chimenea, y alrededor de la mitad carece de drenaje. A partir de la información de la ENIGH se observa también que siete de cada diez viviendas presentan hacinamiento, con un promedio de cuatro personas por dormitorio.

Asimismo, datos del CONEVAL muestran que cerca de la mitad de la población presenta rezago educativo y, además, información de la ENIGH refleja que el 90\% de las mujeres y hombres jefes del hogar en estos municipios no concluyó la primaria, y una tercera parte carece totalmente de instrucción.

La dificultad de acceso a estos municipios y la consecuente ausencia de actividades económicas y alternativas de empleo explican que el ingreso dependa básicamente de los beneficios gubernamentales y de actividades independientes agropecuarias, lo que a su vez explica que prácticamente toda la población carezca de seguridad social (ver Tablas 3 y 5). Ahora bien, aunque parece cubrirse el derecho 
a la salud debido al registro en el Seguro Popular, no necesariamente el acceso al servicio y su calidad son efectivos, por lo que la población recurre principalmente a la medicina tradicional.

Considerando que este conglomerado reúne sólo el $15 \%$ de la población del estado, parece viable sugerir un enfoque de apoyos universalizados para cubrir el acceso a los derechos de alimentación, servicios básicos, calidad y espacios de la vivienda, educación, salud y fomento al empleo agrícola, sobre todo porque la combinación de estas carencias repercute directamente en el desarrollo de capacidades de los habitantes, lo que genera un círculo vicioso de pobreza intergeneracional.

El tipo de apoyo que se considera más conveniente es el de inversión en bienes públicos, por medio de escuelas, comedores comunitarios, centros de salud e infraestructura productiva. También se considera que las transferencias monetarias condicionadas son adecuadas en este conglomerado, sobre todo para cubrir necesidades de alimentación, educación y salud, pero no deben tomarse como la única forma de apoyo pues, de esa manera, lejos de mitigar los niveles de pobreza, únicamente contribuirían a mantener el problema.

\section{Perfil II. Pobreza intensa en las regiones Selva y Frontera}

El perfil II integra 44 municipios que colindan con Guatemala, tanto en la región Selva como en la Frontera (ver Mapa ly Tabla 6). En estas dos regiones se reúne el $26 \%$ de la población del estado, de cinco etnias: zoque, tseltal, tojolabal, mam, mochó y kakchiquel; en algunos de los municipios adscritos a este perfil se concentra la presencia de simpatizantes del Ejército Zapatista de Liberación Nacional.

En cuanto a la dimensión de bienestar, se observa que el ingreso mejora en relación con el perfil I, porque en este caso la población puede cubrir el costo de la canasta básica alimentaria rural, aunque su nivel de bienestar aún es muy bajo para cubrir también el costo de la canasta no alimentaria (ver Tabla 3 ).

Por su parte, el avance en derechos sociales radica principalmente en cierta mejoría en los indicadores de servicios básicos, y calidad y espacios de la vivienda, como consecuencia de más acceso a drenaje, una menor proporción de hogares donde se cocina sin chimenea y un nivel de hacinamiento más bajo. En contraste, este conglomerado agrupa a la población de Chiapas que aún no cuenta con energía eléctrica y presenta mayor proporción de viviendas sin acceso a agua (ver Tabla 4).

Analizando el perfil con información de la ENIGH, se observa que la composición del ingreso se sigue basando en actividades agropecuarias y en beneficios gubernamentales, con una mínima presencia de trabajo asalariado por lo que, en consecuencia, la gran mayoría de la población carece de acceso a la seguridad social (ver Tabla 5). El nivel de rezago educativo medido por el CONEVAL persiste en cuatro de cada diez habitantes de este conglomerado, y más del 80\% de jefes del hogar tiene inconclusa la primaria.

El esquema de política social sugerido en este caso es similar al del primer conglomerado pues implica la necesidad de impulsar el empleo agropecuario y no agropecuario, así como universalizar el acceso a servicios básicos y asegurar el acceso real a los derechos a la educación, la salud y la alimentación.

Sin embargo, es preciso considerar que se trata de un conglomerado que colinda con otro país, que presenta una influencia dominante del Ejército Zapatista de Liberación Nacional y que agrupa a una gran diversidad de etnias. Esta multiculturalidad acentúa la complejidad para hacer coincidir las reglas formales de las instituciones gubernamentales con las reglas informales apropiadas por la población, debido a que, para cubrir derechos sociales, es necesario garantizar los derechos humanos y colectivos.

\section{Perfil III. El hemisferio izquierdo de Chiapas}

El perfil III de esta tipología incluye 34 municipios en los que habita el $27 \%$ de la población del estado. En este caso, la población indígena ya no es predominante, sólo se encuentra presente el grupo chol al noreste del estado. Las entidades que limitan con este conglomerado son Oaxaca, Veracruz y una parte de Tabasco (ver Mapa 1 y Tabla 6). 
A partir de las bases de datos de la ENIGH se observa que en estos municipios se diversifica la composición del ingreso, principalmente porque disminuye considerablemente la aportación de las actividades agrícolas y las transferencias gubernamentales para dar paso al trabajo asalariado y a negocios independientes (ver Tabla 5). No obstante, el ingreso sólo permite cubrir el costo de las canastas alimentarias rural y urbana, no así el de la canasta no alimentaria (ver Tabla 3).

Se observa también que continúa siendo importante el número de viviendas carentes de agua y con cocinas sin chimenea (ver Tabla 4). Asimismo, una proporción elevada de personas no tiene acceso a la educación, a la salud y a la alimentación (ver Tabla 3). Sin embargo, la infraestructura pública permite que las escuelas y centros de salud sean mucho más accesibles. Por otro lado, la falta de acceso a la alimentación resulta ser más un problema de variedad de alimentos, que de cantidad, por lo que no se asocia con el concepto de hambre.

En este sentido, la política propuesta se inclina más a la focalización tanto para los apoyos directos a hogares con carencias en servicios básicos, como en lo que respecta a las transferencias condicionadas destinadas a atender al mismo tiempo alimentación, educación y salud. Por otro lado, al tratarse de municipios aledaños a las principales zonas turísticas y comerciales, se considera que las acciones de política más acertadas serían las dirigidas a mejorar los niveles de ingreso, siendo indispensable impulsar el empleo rural no agrícola, brindar capacitación para la generación de capacidades y facilitar el acceso al crédito.

\section{Perfil IV. Municipios aislados en "las mejores condiciones"}

El último conglomerado es el de carácter más urbano, turístico y heterogéneo, lo que implica una mayor propensión a que las ponderaciones promediales oculten tanto la concentración de la riqueza, como los cinturones de pobreza. En este perfil se incluyen sólo diecisiete municipios (ver Tabla 6), pero en éstos se concentra una tercera parte de la población estatal.
La presencia indígena es dispersa en la mayoría de los municipios incluidos en este perfil, con excepción de San Cristóbal de Las Casas. Este conglomerado se ubica en parte de las regiones Centro, Istmo-Costa, Norte y Soconusco (ver Mapa ly Tabla 4).

El ingreso promedio en este perfil se encuentra por encima del costo de las canastas alimentaria y no alimentaria rurales; sin embargo, ni siquiera en este conglomerado se alcanza el nivel de bienestar del ámbito urbano, lo que confirma que en la entidad existe una situación generalizada deingresos muy bajos (ver Tabla3).

Los derechos sociales que muestran mayor avance son la calidad y los espacios de la vivienda, la alimentación y la educación, situación que se explica por el nivel de urbanización del conglomerado. Por otro lado, y aun tratándose de zonas urbanas, cerca de la mitad de la población no tiene acceso a servicios básicos, y una tercera parte carece de acceso a servicios de salud (ver Tablas 3 y 4 ).

$\mathrm{Al}$ analizar este perfil con los microdatos de la ENIGH, se observa que en él se ubica un mayor número de pobladores cuyos ingresos dependen más del trabajo asalariado y de negocios independientes (ver Tabla 5). Sin embargo, aun en este perfil el $73 \%$ de la población carece de acceso a la seguridad social debido a que en la entidad predomina el empleo informal.

Es importante recalcar que el nivel de heterogeneidad de este perfil implica mayor necesidad de una agenda pública que focalice los apoyos y establezca las normas y procedimientos necesarios para llegar a la población que verdaderamente los necesita, principalmente para cubrir las carencias de acceso a servicios básicos y de salud.

Ahora bien, para este tipo de municipios, donde se cuenta con mayor avance en el ámbito de derechos sociales pero persiste un nivel de ingresos muy bajo para cubrir las necesidades de las familias, las políticas públicas deben prestar especial atención a la promoción de la educación superior, la innovación, la generación de empresas, el acceso al crédito, la capacitación para microempresarios y la generación de empleos formales que permitan acceder a la seguridad social. 


\section{Discusión}

Un objetivo central en todos los países es, o debería ser, mejorar el nivel de bienestar de la totalidad de su población, por un lado permitiendo el pleno ejercicio de sus derechos sociales y, por otro, facilitando que todas las personas cuenten con la capacidad económica para satisfacer sus necesidades presentes y futuras. Para lograrlo, los comparativos de pobreza que permiten observar dónde o cuándo la pobreza es mayor deberían tener un peso más significativo en las decisiones políticas que las medidas agregadas (Ravallion y Bidani, 1993: 98).

Si bien es cierto que aun en los perfiles descritos se esconden desigualdades y particularidades inherentes a la utilización de promedios, la caracterización de cada uno de ellos ofrece una visión más amplia y más fácil de visualizar geográficamente que puede ayudar en la búsqueda de alternativas para superar la pobreza en Chiapas. Por ejemplo, los perfiles I y II coinciden con hallazgos recientes de otros autores que han encontrado que la agudización de la pobreza en las comunidades indígenas y en algunos municipios con alto nivel de marginación llevó al levantamiento en armas del Ejército Zapatista de Liberación Nacional (Ávila, Sulvarán y Ávila, 2014; Villafuerte, 2015; Aguilar, 2016).

La necesidad emergente de implementar un proyecto a largo plazo y de universalizar el acceso a los derechos sociales en los municipios del perfil I coincide también con los hallazgos de Martínez y Fletes (2015), quienes en su investigación mostraron que la región de Los Altos de Chiapas presenta los niveles de pobreza más elevados, pero que, además, ésta se ha venido incrementando durante los últimos años.

Ahora bien, en las políticas propuestas se insiste en la necesidad de analizar la intensidad de la pobreza multidimensional en los municipios para elegir entre universalización y focalización de programas (Brodersohn, 1999). Por ejemplo, los indicadores muy elevados de pobreza en los perfiles I y II muestran que los programas de apoyo focalizados tienen la gran desventaja de abordar de manera muy superficial, e incluso discrecional, problemas que son estructurales y generalizados.
A partir del perfil III la universalización resulta menos viable dada la densidad de la población, por lo que se plantea cuidar la focalización de los apoyos a las familias más vulnerables, sin dejar de lado la provisión de bienes públicos principalmente para cubrir el acceso a servicios básicos, educación y salud.

Para impulsar la dimensión de bienestar, en los municipios ubicados en todos los perfiles se requiere de la generación de capacidades para responder a las posibilidades de los contextos locales, es decir, se observa la necesidad de fomentar la capacitación y el apoyo a la inversión y comercialización en actividades que ya se desarrollan en los municipios como la producción agrícola, pecuaria, artesanal o turística. En este sentido, se sugiere evitar limitarse a las transferencias condicionadas y apostar más por el fomento productivo, rubro al que se han asignado mínimos recursos en los últimos años en la composición del presupuesto estatal (Villafuerte, 2015).

Finalmente, es necesario reconocer que la pobreza en Chiapas es un fenómeno multicausal y muy complejo, por lo que las políticas aquí descritas se alinean a las dimensiones de bienestar y derechos sociales que se consideran en la actual medición multidimensional de la pobreza, pero no necesariamente son las únicas o las mejores.

\section{Conclusiones}

Los perfiles que se obtuvieron en este trabajo evidencian la intensidad de la pobreza multidimensional en Chiapas, pero también la heterogeneidad cuando el análisis se realiza a nivel municipal, lo que ofrece un panorama que se considera novedoso y útil en el diseño de políticas públicas.

Chiapas debe mantenerse como una entidad prioritaria para hacer frente al tema de la pobreza, pero la estrategia debe transitar de la atención a la erradicación. En este sentido, y considerando que el problema es mayor en donde es más intenso y generalizado, los municipios que integran los perfiles I y II deben ser prioritarios. 
El esquema de apoyos que se implemente en cada municipio debe ser congruente con el nivel de urgencia de la población, con las principales necesidades de los hogares y con el potencial productivo de la región, además de que debe tomarse en cuenta el contexto geográfico y cultural. En este sentido, esquemas de apoyo como las transferencias condicionadas se consideran válidos, e incluso necesarios, para una parte de la población de Chiapas durante un período de tiempo. Sin embargo, el fomento al empleo es la estrategia más eficaz para brindar a los hogares autosuficiencia para mejorar sus viviendas, el acceso a la alimentación, la educación, la salud y el nivel de bienestar en general.

\section{Referencias}

Aguilar, Teodoro (2016). "Desigualdad y marginación en Chiapas”. En Península, XI(2): 133-149.

Aldenderfer, Mark y Roger Blashfield (1984). Cluster Analysis. Beverly Hills: Sage Publications.

Alkire, Sabina, Adriana Conconi y Suman Seth (2014). "Multidimensional Poverty Index 2014: Brief Methodological Note and Results". Oxford, Reino Unido: University of Oxford. MPI 2014. Methodological Note.

Alkire, Sabina y James Foster (2011). "Understandings and misunderstandings of multidimensional poverty measurement". En The Journal of Economic Inequality, 9(2): 289-314.

Alemayehu, Geda, Niek de Jong, Mwangi S. Kimenyi y Germano Mwabu (2005). "Determinants of Poverty in Kenya: A Household Level Analysis". Working Paper 2005-44. Storrs: University of Connecticut. Department of Economics Working Paper Series, enero.

Ávila, Agustín, José Luis Sulvarán y León Ávila (2014). "El estado mexicano de Chiapas: ¿Qué ha cambiado en veinte años?" En Apuntes del CENES, 33(58): 135-162.

Backiny-Yetna, Prospere y Quentin Wodon (2009). "Profil et perceptions de la pauvreté en République du Congo en 2005". En Perspective Afrique, 4(1-3).

Brodersohn, Víctor (1999). "Focalización de programas de superación de la pobreza”. En Derecho a tener derecho: infancia, derecho y políticas sociales en América Latina. Santiago de Chile: Instituto Interamericano del Niño, UNICEF.

CONEVAL (Consejo Nacional de Evaluación de la Política de Desarrollo Social) (2009). Metodología para la medición multidimensional de la pobreza en México. México: CONEVAL.

CONEVAL (Consejo Nacional de Evaluación de la Política de Desarrollo Social) (2010). Estimaciones del CONEVAL con base en el MCS-ENIGH 2010 y la muestra del Censo de Población y Vivienda 2010. México: CONEVAL.

CONEVAL (Consejo Nacional de Evaluación de la Política de Desarrollo Social) (2017). Resultados de pobreza en México 2016 a nivel nacional y por entidades federativas. México: CONEVAL.

Cortés, Fernando, Israel Banegas, Tabaré Fernández y Minor Mora (2007). "Perfiles de pobreza en Chiapas". En Sociológica, 22(63): 19-50.

Dresser, Denise (1997). "En busca de la legitimidad perdida: PRONASOL, pobreza y política en el gobierno de Salinas". En G. Martínez (comp), Pobreza y política social en México. México: ITAM, Fondo de Cultura Económica, pp. 21-52.

Gordon, David (2004). "La medición internacional de la pobreza y las políticas para combatirla". En J. Boltvinik y A. Damián (coords.), La pobreza en México y el mundo: realidades y desafíos. México: Siglo XXI Editores.

Lerner, Bertha (1996). América Latina: los debates en política social, desigualdad y pobreza. México: Porrúa.

Martínez, Juana y Héctor B. Fletes (2015). “La persistencia de la pobreza en los altos de Chiapas. Factores estructurales y regionales". Ponencia presentada en el 20 Encuentro Nacional sobre Desarrollo Regional en México, AMECIDER-CRIM, UNAM.

Montanero, Jesús (2008). Análisis multivariante. Cáceres: Universidad de Extremadura.

Olinto, Pedro, Kathleen Beegle, Carlos Sobrado e Hiroki Uematsu (2013). "The State of the Poor: Where Are the Poor, Where is Extreme Poverty Harder to End, and What is the Current Profile of the World's Poor?" En The World Bank. Economic Premise, 125, octubre. Poverty Reduction and Economic Management Network (PREM). 
Peña, Daniel (2002). Análisis de datos multivariantes. Madrid: Mc-Graw Hill.

Ravallion, Martin y Benu Bidani (1993). “iHow Robust is a Poverty Profile?" En The World Bank Economic Review, 8(1): 75-102.

Rodríguez, Luis (2007) "Del asistencialismo a la subsidiaridad: una política de atención a la pobreza". En R. Cordera y C. Cabrera (coord.), La política social en México: tendencias y perspectivas. México: Facultad de Economía, Universidad Nacional Autónoma de México.
Sen, Amartya (1991). "Welfare, Preference and Freedom". En Journal of Econometrics, 50(1-2): 15-29.

Sen, Amartya (2003). "Development as Capability Expansion". En Sakiko Fukuda-Parr y Shiva Kumar (eds.), Readings in Human Development. Nueva York: Oxford University Press, pp. 3-16.

Townsend, Peter (1993). The International Analysis of Poverty. Nueva York: Routledge.

Villafuerte Solís, Daniel (2015). "Crisis rural, pobreza y hambre en Chiapas". En LiminaR. Estudios Sociales y Humanísticos, XIII(1): 13-28. 
Figura 1. Indicadores de pobreza multidimensional en México y Chiapas

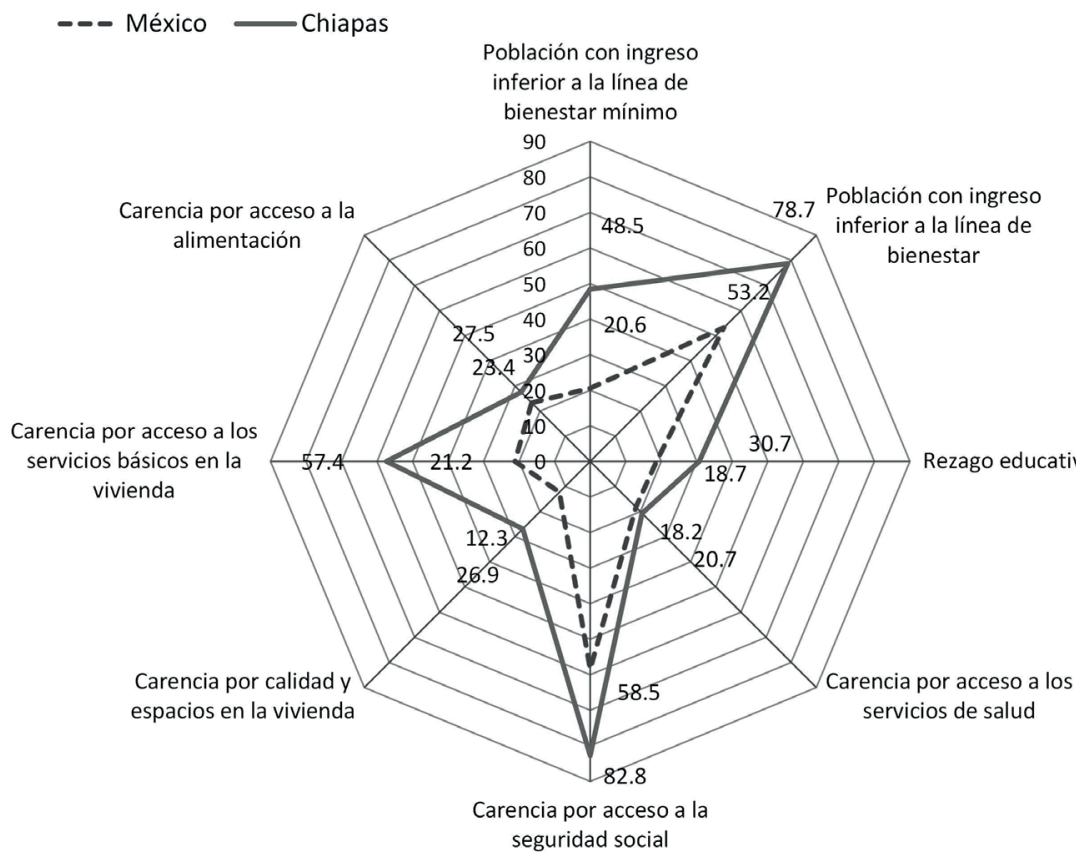

Fuente: elaboración propia con base en estadísticas del CONEVAL.

Mapa 1. Perfiles de pobreza multidimensional en Chiapas

\section{Clusters}

municipal

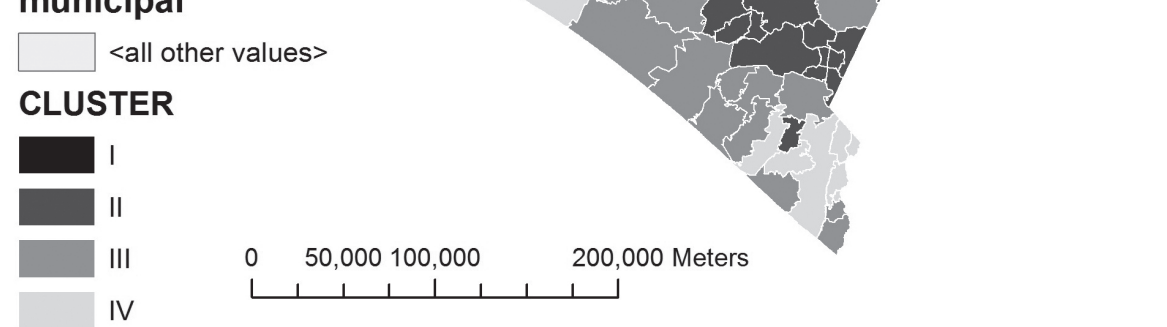

Fuente: elaboración propia con base en resultados y el Mapa Geoestadístico Nacional 2010 del INEGI. 
Tabla 1. Análisis de varianza univariados

\begin{tabular}{crrrrrrr}
\hline Variable & \multicolumn{1}{c}{ SC Trat } & gl Grupo & CM Grupo & CM Error & gl Error & F & p-valor \\
\hline ING & 14781014,38 & 3 & 4927004,79 & 17756,87 & 114 & 277,47 & $<0,0001$ \\
SBV & 2,76 & 3 & 0,92 & 0,02 & 114 & 59,53 & $<0,0001$ \\
VIV & 1,14 & 3 & 0,38 & 0,01 & 114 & 46,15 & $<0,0001$ \\
ALI & 0,48 & 3 & 0,16 & $3,5 \mathrm{E}-03$ & 114 & 45,63 & $<0,0001$ \\
EDU & 0,46 & 3 & 0,15 & $2,6 \mathrm{E}-03$ & 114 & 60,24 & $<0,0001$ \\
SAL & 0,02 & 3 & 0,01 & 0,03 & 114 & 0,26 & 0,8555 \\
SSO & 0,58 & 3 & 0,19 & $4,1 \mathrm{E}-03$ & 114 & 46,48 & $<0,0001$ \\
\hline
\end{tabular}

Fuente: resultados obtenidos con el software estadístico Infostat.

Tabla 2. Prueba de homogeneidad de matrices de covarianzas

\begin{tabular}{ccccc}
\hline Grupos & N & Estadístico & gl & p-valor \\
\hline 4 & 118 & 571,86 & 84 & $<0,0001$ \\
\hline
\end{tabular}

Fuente: resultados obtenidos con el software estadístico Infostat.

Tabla 3. Heterogeneidad de ingreso y carencias sociales entre perfiles

\begin{tabular}{|c|c|c|c|c|c|}
\hline \multicolumn{2}{|c|}{ Indicador / Perfil } & I & II & III & IV \\
\hline \multicolumn{2}{|c|}{ Número de municipios } & 23 & 44 & 34 & 17 \\
\hline \multirow[t]{3}{*}{ Bienestar económico } & Ingreso promedio per cápita mensual & $\$ 569$ & $\$ 805$ & $\$ 1,191$ & $\$ 1,832$ \\
\hline & Servicios básicos & $92 \%$ & $70 \%$ & $56 \%$ & $45 \%$ \\
\hline & Calidad y espacios de la vivienda & $55 \%$ & $35 \%$ & $33 \%$ & $24 \%$ \\
\hline \multirow{4}{*}{$\begin{array}{l}\text { Derechos sociales } \\
\text { (Porcentaje promedio de } \\
\text { carencia por acceso) }\end{array}$} & Alimentación & $44 \%$ & $32 \%$ & $30 \%$ & $23 \%$ \\
\hline & Rezago educativo & $46 \%$ & $41 \%$ & $36 \%$ & $26 \%$ \\
\hline & Servicios de salud & $32 \%$ & $32 \%$ & $35 \%$ & $33 \%$ \\
\hline & Seguridad social & $97 \%$ & $89 \%$ & $87 \%$ & $73 \%$ \\
\hline
\end{tabular}

Fuente: elaboración propia con base en resultados.

Línea de bienestar mínimo rural: \$684 Línea de bienestar mínimo urbana: \$978

Línea de bienestar rural: \$1,330 Línea de bienestar urbana: \$2,120

* Líneas de bienestar correspondientes al momento del levantamiento de información del MCS-ENIGH 2010. 
Tabla 4. Comparativo de servicios básicos y calidad y espacios de la vivienda

\begin{tabular}{|c|c|c|c|c|}
\hline Indicador / Perfil & \multirow{2}{*}{ I } & \multirow{2}{*}{ II } & \multirow{2}{*}{ III } & \multirow{2}{*}{ IV } \\
\hline Carencia por acceso a servicios básicos en la vivienda & & & & \\
\hline Viviendas sin acceso al agua & $19 \%$ & $23 \%$ & $27 \%$ & $18 \%$ \\
\hline Viviendas sin drenaje & $46 \%$ & $35 \%$ & $12 \%$ & $8 \%$ \\
\hline Viviendas sin electricidad & $0.0 \%$ & $5.4 \%$ & $0.4 \%$ & $0.3 \%$ \\
\hline Viviendas sin chimenea cuando usan leña o carbón para cocinar & $87 \%$ & $62 \%$ & $41 \%$ & $27 \%$ \\
\hline \multicolumn{5}{|l|}{ Carencia por acceso a calidad y espacios de la vivienda } \\
\hline Viviendas con pisos de tierra & $13 \%$ & $13 \%$ & $4 \%$ & $7 \%$ \\
\hline Viviendas con techos de material endeble & $0.6 \%$ & $0.8 \%$ & $0.6 \%$ & $0.6 \%$ \\
\hline Viviendas con muros de material endeble & $5 \%$ & $1 \%$ & $6 \%$ & $6 \%$ \\
\hline Viviendas con hacinamiento & $72 \%$ & $59 \%$ & $50 \%$ & $37 \%$ \\
\hline Promedio de personas por cuarto & 4.1 & 3.1 & 2.8 & 2.3 \\
\hline
\end{tabular}

Fuente: elaboración propia con base en resultados y MCS-ENIGH 2010.

Tabla 5. Comparativo de composición del ingreso en los hogares

\begin{tabular}{lcccc}
\hline \multicolumn{1}{c}{ Fuente de ingreso/ Perfil } & I & II & III & IV \\
\hline Trabajo asalariado & $15 \%$ & $15 \%$ & $41 \%$ & $55 \%$ \\
Negocios agropecuarios & $32 \%$ & $29 \%$ & $10 \%$ & $4 \%$ \\
Negocios no agropecuarios & $4 \%$ & $5 \%$ & $15 \%$ & $12 \%$ \\
Otros trabajos & $6 \%$ & $5 \%$ & $5 \%$ & $5 \%$ \\
Rentas & $0 \%$ & $1 \%$ & $1 \%$ & $2 \%$ \\
Jubilación & $0 \%$ & $1 \%$ & $2 \%$ & $5 \%$ \\
Becas & $0 \%$ & $0 \%$ & $0 \%$ & $0 \%$ \\
Donativos & $3 \%$ & $2 \%$ & $2 \%$ & $5 \%$ \\
Remesas & $0 \%$ & $1 \%$ & $1 \%$ & $0 \%$ \\
Beneficios gubernamentales & $35 \%$ & $39 \%$ & $19 \%$ & $9 \%$ \\
Transferencias de hogares & $2 \%$ & $1 \%$ & $2 \%$ & $3 \%$ \\
Transferencias de instituciones & $2 \%$ & $1 \%$ & $0 \%$ & $0 \%$ \\
Otros ingresos & $0 \%$ & $0 \%$ & $0 \%$ \\
\hline
\end{tabular}

Fuente: elaboración propia con base en resultados y MCS-ENIGH 2010. 
Tabla 6. Municipios de Chiapas en cada perfil de pobreza multidimensional

\begin{tabular}{|c|c|}
\hline $\begin{array}{l}\text { Municipios } \\
\text { tipo I }\end{array}$ & $\begin{array}{l}\text { Amatenango del Valle, Chalchihuitán, Chamula, Chanal, Chenalhó, Chilón, Huixtán, Larráinzar, } \\
\text { Mitontic, Ocotepec, Oxchuc, Pantelhó, Sabanilla, Sitalá, Tenejapa, Tila, Tumbalá, San Lucas, } \\
\text { Zinacantán, San Juan Cancuc, Aldama, Maravilla Tenejapa, Santiago el Pinar. }\end{array}$ \\
\hline $\begin{array}{l}\text { Municipios } \\
\text { tipo II }\end{array}$ & $\begin{array}{l}\text { Altamirano, Amatán, Amatenango de la Frontera, Ángel Albino Corzo, Bejucal de Ocampo, Bella } \\
\text { Vista, El Bosque, Coapilla, Chapultenango, Chiapilla, Chicomuselo, Francisco León, La Grandeza, } \\
\text { Huitiupán, La Independencia, Ixhuatán, Ixtapa, Ixtapangajoya, Jitotol, Las Margaritas, Mazapa } \\
\text { de Madero, Nicolás Ruiz, Ocosingo, Osumacinta, Pantepec, El Porvenir, Pueblo Nuevo } \\
\text { Solistahuacán, Rayón, Salto de Agua, Siltepec, Simojovel, Socoltenango, Solosuchiapa, Sunuapa, } \\
\text { Tapalapa, Teopisca, Totolapa, La Trinitaria, Tuzantán, Tzimol, Benemérito de las Américas, } \\
\text { Marqués de Comillas, Montecristo de Guerrero, San Andrés Duraznal. }\end{array}$ \\
\hline $\begin{array}{l}\text { Municipios } \\
\text { tipo III }\end{array}$ & $\begin{array}{l}\text { Acacoyagua, Acala, Acapetahua, Berriozábal, Bochil, Catazajá, Cintalapa, La Concordia, } \\
\text { Copainalá, Escuintla, Frontera Comalapa, Frontera Hidalgo, Ixtacomitán, Juárez, La Libertad, } \\
\text { Mapastepec, Mazatán, Motozintla, Ocozocoautla de Espinosa, Ostuacán, Palenque, Pijijiapan, } \\
\text { Villa Comaltitlán, Las Rosas, San Fernando, Soyaló, Suchiapa, Suchiate, Tapilula, Tecpatán, } \\
\text { Venustiano Carranza, Villa Corzo, Villaflores, Yajalón. }\end{array}$ \\
\hline $\begin{array}{l}\text { Municipios } \\
\text { tipo IV }\end{array}$ & $\begin{array}{l}\text { Arriaga, Cacahoatán, Comitán de Domínguez, Chiapa de Corzo, Chicoasén, Huehuetán, Huixtla, } \\
\text { Jiquipilas, Metapa, Pichucalco, Reforma, San Cristóbal de Las Casas, Tapachula, Tonalá, Tuxtla } \\
\text { Gutiérrez, Tuxtla Chico, Unión Juárez. }\end{array}$ \\
\hline
\end{tabular}

Fuente: elaboración propia con base en resultados. 